\title{
OpenJIT: An Open-Ended, Reflective JIT Compile Framework for Java
}

\author{
Hirotaka Ogawa ${ }^{1}$, Kouya Shimura ${ }^{2}$, Satoshi Matsuoka ${ }^{1}$, Fuyuhiko Maruyama $^{1}$, Yukihiko \\ Sohda ${ }^{1}$, and Yasunori Kimura ${ }^{2}$ \\ 1 Tokyo Institute of Technology \\ 2 Fujitsu Laboratories Limited
}

\begin{abstract}
OpenJIT is an open-ended, reflective JIT compiler framework for Java being researched and developed in a joint project by Tokyo Inst. Tech. and Fujitsu Ltd. Although in general self-descriptive systems have been studied in various contexts such as reflection and interpreter/compiler bootstrapping, OpenJIT is a first system we know to date that offers a stable, full-fledged Java JIT compiler that plugs into existing monolithic JVMs, and offer competitive performance to JITs typically written in $\mathrm{C}$ or $\mathrm{C}++$. This is in contrast to previous work where compilation did not occur in the execution phase, customized VMs being developed ground-up, performance not competing with existing optimizing JIT compilers, and/or only a subset of the Java language being supported. The main contributions of this paper are, 1) we propose an architecture for a reflective JIT compiler on a monolithic VM, and identify the technical challenges as well as the techniques employed, 2) We define an API that adds to the existing JIT compiler APIs in "classic" JVM to allow reflective JITs to be constructed, 3) We show detailed benchmarks of run-time behavior of OpenJIT to demonstrate that, while being competitive with existing JITs the time- and space-overheads of compiler metaobjects that exist in the heap are small and manageable, and 4) we demonstrate how reflective JITs could be useful class- or application specific customization and optimization by providing an important reflective "hook" into a Java system. Being an object-oriented compiler framework, OpenJIT can be configured to be small and portable or fully-fledged optimizing compiler framework in the spirit of SUIF. It is fully JCK compliant, and runs all large Java applications we have tested to date including HotJava. We are currently distributing OpenJIT for free to foster further research into advanced compiler optimization, compile-time reflection, advanced run-time support for languages, as well as other areas such as embedded computing, metacomputing, and ubiquitous computing.
\end{abstract}

\section{Introduction}

Programming Languages with high-degree of portability, such as Java, typically employ portable intermediate program representations such as bytecodes, and utilize Just-In-Time compilers (JITs), which compile (parts of) programs into native code at runtime. However, all the Java JITs today as well as those for other languages such as Lisp, Smalltalk, and Self, only largely focuses on standard platforms such as Workstations and PCs, merely stress optimizing for speeding up single-threaded execution of general programs, usually at the expense of memory for space-time tradeoff. This is not appropriate, for example, for embedded systems where the tradeoff should be shifted more to memory rather than speed. Moreover, we claim that JITs could be utilized and exploited more opportunely in the following situations:

- Platform-specific optimizations: Execution platforms could be from embedded systems and hand-held devices all the way up to large servers and massive parallel processors (MPPs). There, requirements for optimizations differ considerably, not only for space-time 
tradeoffs, but also for particular class of applications that the platform is targeted to execute. JITs could be made to adapt to different platforms if it could be customized in a flexible way.

- Platform-specific compilations: On related terms, some platforms require assistance of compilers to generate platform- specific codes for execution. For example, DSM (Distributed-Shared Memory) systems and persistent object systems require specific compilations to emit code to detect remote or persistent reference operations. Thus, if one were to implement such systems on Java, one not only needs to modify the JVM, but also the JIT compiler. We note that, as far as we know, representative work on Java DSM (cJVM[AFT99] by IBM) and persistent objects (PJama[ADJ+96] at University of Glasgow) lack JIT compiler support for this very reason.

- Application-specific optimizations: One could be more opportunistic by performing optimizations that are specific to a particular application or a data set. This includes techniques such as selection of compilation strategies, runtime partial evaluation, as well as application-specific idiom recognition. By utilizing application-specific as well as runtime information, the compiled code could be made to execute substantially faster, or with less space, etc. compared to traditional, generalized optimizations. Although such techniques have been proposed in the past, it could become a generally-applied scheme and also an exciting research area if efficient and easily customizable JITs were available.

- Language-extending compilations: Some work stresses on extending Java for adding new language features and abstractions. Such extensions could be implemented as sourcelevel or byte-code level transformations, but some low-level implementations are very difficult or inefficient to support with such higher-level transformations in Java. The abovementioned DSM is a good example: Some DSMs permit users to add control directives or storage classifiers at a program level to control the memory coherency protocols, and thus such a change must be done at JVM and native code level. One could facilitate this by encoding such extensions in bytecodes or classfile attributes, and customizing the JIT compilers accordingly to understand such extensions.

- Environment- or Usage-specific compilations and optimizations: Other environmental or usage factors could be considered during compilation, such as adding profiling code for performance instrumentation, debugging etc. ${ }^{1}$

Moreover, with Java, we would like these customizations to occur within an easy framework of portable, security-checked code downloaded across the network. That is to say, just as applets and libraries are downloadable on-the-fly, we would like the JIT compiler customization to be downloaded on-the-fly as well, depending on the specific platform, application, and environment. For example, if a user wants to instrument his code, he will want to download the (trusted) instrumentation component from the network on-the-fly to customize the generated code accordingly.

Unfortunately, most JITs today, especially those for Java, are architected to be closed and monolithic, and do not facilitate interfaces, frameworks, nor patterns as a means of customization. Moreover, JIT compilers are usually written in $\mathrm{C}$ or $\mathrm{C}++$, and live in a completely separate scope from normal Java programs, without enjoying any of the language/systems benefits that Java provides, such as ease of programming and debugging, code safety, portability and mobility, etc. In other words, current Java JIT compilers are "black boxes", being in a sense against the principle of modular, open-ended, portable design ideals that Java itself represents.

In order to resolve such a situation, the collaborative group between Tokyo Institute of Technology and Fujitsu Limited have been working on a project OpenJIT[MOS ${ }^{+}$98] for almost

\footnotetext{
${ }^{1}$ In fact we do exactly that in the benchmarking we show later in Section 5, which for the first time characterizes the behavior of a self-descriptive JIT compiler.
} 
the past two years. OpenJIT itself is a "reflective" Just-In-Time open compiler framework for Java written almost entirely in Java itself, and plugs into the standard Sun JDK 1.1.x and 1.2 JVMs. All compiler objects coexist in the same heap space as the application objects, and are subject to execution by the same Java machinery, including having to be compiled by itself, and subject to static and dynamic customizations. At the same time, it is a fullyfledged, JCK (Java Compatibility Kit) compliant JIT compiler, able to run production Java code. In fact, as far as we know, it is the ONLY Java JIT compiler whose source code is available in public, and is JCK compliant other than that of Sun's. And, as the benchmarks will show, although being constrained by the limitations of the "classic" JVMs, and still being in development stage lacking sophisticated high-level optimizations, it is nonetheless equal to or superior to the Sun's (classic) JIT compiler on SpecJVM benchmarks, and attains about half the speed of the fastest JIT compilers that are much more complex, closed, and requires a specialized JVM. At the same time, OpenJIT is designed to be a compiler framework in the sense of Stanford SUIF[Uni], in that it facilitates high-level and low-level program analysis and transformation framework for the users to customize.

OpenJIT is still in active development, and we have just started distributing it for free for non-commercial purposes from http://www.openjit.org/. It has shown to be quite portable, thanks in part to being written in Java-the Sparc version of OpenJIT runs on Solaris, and the x86 version runs on different breeds of Unix including Linux, FreeBSD, and Solaris. We are hoping that it will stem and cultivate interesting and new research in the field of compiler development, reflection, portable code, language design, dynamic optimization, and other areas.

The purpose of the paper is to describe our experiences in building OpenJIT, as well as presenting the following technical contributions:

1. We propose an architecture for a reflective JIT compiler framework on a monolithic "classic" JVM, and identify the technical challenges as well as the techniques employed. The challenges exist for several reasons, that the JIT compiler is reflective, and also the characteristics of Java, such as its pointer-safe execution model, built-in multi-threading, etc.

2. We show an API that adds to the existing JIT compiler APIs in "classic" JVM to allow reflective JITs to be constructed. Although still early in its design, and requiring definitions of higher-level abstractions as well as additional APIs for supporting JITs on more modern VMs, we nonetheless present a minimal set of APIs that were necessary to be added to the Java VM in order to facilitate a Java JIT compiler in Java.

3. We perform extensive analysis of the performance characteristics of OpenJIT, both in terms of execution speed and memory consumption. In fact, as far as we know, there have not been any reports on any self-descriptive JIT compilation performance analysis, nor memory consumption reports for any JIT compilers. In particular, we show that (1) JIT compilation speed does not become a performance issue, especially during the bootstrap process when much of the OpenJIT compiler is run under interpretation, (2) memory consumption of reflective JITs, however, could be problematic due to recursive compilation, especially in embedded situations, (3) that there are effective strategies to solve the problems, which we investigate extensively, and (4) that the solutions do not add significant overhead to overall execution, due to (1). In fact, the self-compilation time of OpenJIT is quite amortizable for real applications.

4. We demonstrate how reflective JITs could be useful class- or application specific customization and optimization by providing an important reflective "hook" into a Java system, with the notion of compilets. Although the current examples are small, we nevertheless present a possibility of larger-scale deployment of OpenJIT for uses in the abovementioned situations. 


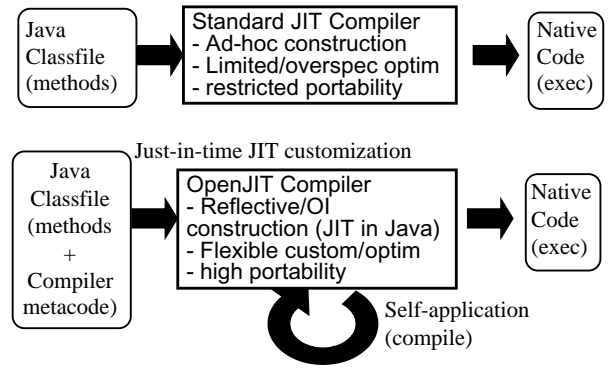

Fig. 1. Comparison of Traditional JITs and OpenJIT

\section{Overview of the OpenJIT Framework}

\subsection{OpenJIT: The Conceptual Overview}

OpenJIT is a JIT compiler written in Java to be executed on "classic" VM systems such as Sun JDK 1.1.x and JDK 1.2.x. OpenJIT allows a given Java code to be portable and maintainable with compiler customization. With standard Java, the portability of Java is effective insofar as the capabilities and features provided by the JVM (Java Virtual Machine); thus, any new features that has to be transparent from the Java source code, but which JVM does not provide, could only be implemented via non-portable means. For example, if one wishes to write a portable parallel application under multi-threaded, shared memory model, then some form of distributed shared memory (DSM) would be required for execution under MPP and cluster platforms. However, JVM itself does not facilitate any DSM functionalities, nor provide any software 'hooks' for incorporating the necessary read/write barriers for userlevel DSM implementation. As a result, one must either modify the JVM, or employ some ad-hoc preprocessor solution, neither of which are satisfactory in terms of portability and/or performance. With OpenJIT, the DSM class library implementor can write a set of compiler metaclasses so that necessary read/write barriers, etc., would be appropriately inserted into critical parts of code.

Also, with OpenJIT, one could incorporate platform-, application-, or usage-specific compilation or optimization. For example, one could perform various numerical optimizations such as loop restructuring, cache blocking, etc. which have been well-studied in Fortran and C, but have not been well adopted into JITs for excessive runtime compilation cost. OpenJIT allows application of such optimizations to critical parts of code in a pinpointed fashion, specified by either the class-library builder, application writer, or the user of the program. Furthermore, it allows optimizations that are too application and/or domain specific to be incorporated as a general optimization technique for standard compilers, as has been reported by [KLM $\left.{ }^{+} 97\right]$.

In this manner, OpenJIT allows a new style of programming for optimizations, portability, and maintainability, compared to traditional JIT compilers, by providing separations of concerns with respect to optimization and code-generation for new features. That is to say, with traditional JIT compilers, we see in the upper half of Figure 1, the JIT compilers would largely be transparent from the user, and users would have to maintain code which might not be tangled to achieve portability and performance. OpenJIT, on the other hand, will allow the users to write clean code describing the base algorithm and features, and by selecting the appropriate compiler metaclasses, or even by writing his own separately, one could achieve optimization while maintaining appropriate separation of concerns. Furthermore, compared to previous open compiler efforts, OpenJIT could achieve better portability and performance, as source code is not necessary, and late binding at run-time allows exploitation of run-time values, as is with run-time code generators. 


\subsection{Architectural Overview of OpenJIT}

The OpenJIT architecture is largely divided into the frontend and the backend processors. The frontend takes the Java bytecodes as input, performs higher-level optimizations involving source-to-source transformations, and passes on the intermediate code to the backend, or outputs the transformed bytecode. The backend is effectively a small JIT compiler in itself, and takes either the bytecode or the intermediate code from the frontend as input, performs lower-level optimizations including transformation to register code, and outputs the native code for direct execution. The reason why there is a separate frontend and the backend is largely due to modularity and ease of development, especially for higher-level transformations, as well as defaulting to the backend when execution speed is not of premium concern. In particular, we strive for the possibility of the two modules being able to run as independent components.

Upon invocation, the OpenJIT frontend system processes the bytecode of the method in the following way: The decompiler recovers the AST of the original Java source from the bytecode, by recreating the control-flow graph of the source program. At the same time, the annotation analysis module will obtain any annotating info on the class file, which will be recorded as attribute info on the $\mathrm{AST}^{2}$.

Next, the obtained AST will be subject to optimization by the (higher-level) optimization module. Based on the AST and control-flow information, we compute the data \& control dependency graphs, etc., and perform program transformation in a standard way with modules such as flowgraph construction module, program analysis module, and program transformation module using template matching. The result from the OpenJIT frontend will be a new bytecode stream, which would be output to a file for later usage, or an intermediate representation to be used directly by the OpenJIT backend.

The OpenJIT backend system, in turn, performs lower-level optimization over the output from the frontend system, or the bytecodes directly, and generates native code. It is in essence a small JIT compiler in itself.

Firstly, when invoked as an independent JIT compiler bypassing the frontend, the low-level IL translator analyzes and translates the bytecode instruction streams to low-level intermediate code representation using stacks. Otherwise the IL from the frontend is utilized. Then, the RTL Translator translates the stack-based code to intermediate code using registers (RTL). Here, the bytecode is analyzed to divide the instruction stream into basic blocks, and by calculating the depth of the stack for each bytecode instruction, the operands are generated with assumption that we have infinite number of registers. Then, the peephole optimizer would eliminate redundant instructions from the RTL instruction stream, and finally, the native code generator would generate the target code of the CPU, allocating physical registers. Currently, OpenJIT supports the SPARC and the x 86 processors as the target, but could be easily ported to other machines. The generated native code will be then invoked by the Java VM, as described earlier.

\section{Details of the OpenJIT Frontend System}

As described in Section 2, the OpenJIT frontend system provides a Java class framework for higher-level, abstract analysis, transformation, and specialization of Java programs which had already been compiled by javac: (1) The decompiler translates the bytecode into augmented AST, (2) analysis, optimizations, and specialization are performed on the tree, and (3) the AST is converted into the low-level IL of the backend system, or optionally, a stream of bytecodes is generated.

\footnotetext{
${ }^{2}$ In the current implementation, the existence of annotation is a prerequisite for frontend processing; otherwise, the frontend is bypassed, and the backend is invoked immediately.
} 


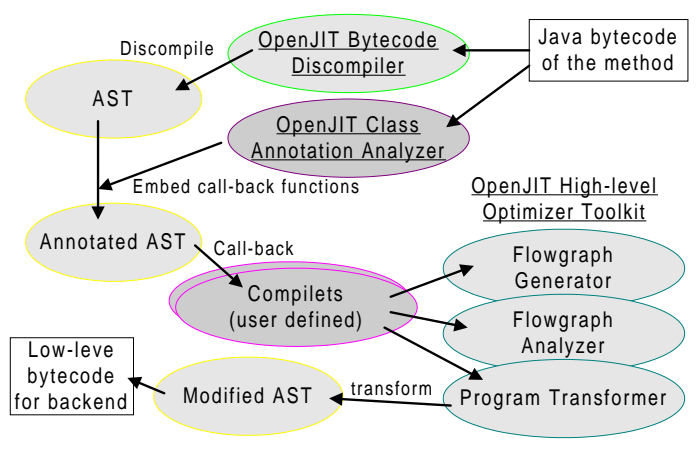

Fig. 2. Overview of OpenJIT Frontend System

Transformation over AST is done in a similar manner to Stanford SUIF, in that there is a method which traverses the tree and performs update on a node or a subtree when necessary. There are a set of abstract methods that are invoked as a hook. The OpenJIT frontend system, in order to utilize such a hook functionality according to user requirements, extends the class file (albeit in a conformable way so that it is compatible with other Java platforms) by adding annotation info to the classfile. Such an info is called "classfile annotation".

The overall architecture of the OpenJIT frontend system is as illustrated in Fig. 2, and consists of the following four modules:

\section{OpenJIT Bytecode Decompiler}

Translates the bytecode stream into augmented AST. It utilizes a new algorithm for systematic AST reconstruction using dominator trees.

2. OpenJIT Class Annotation Analyzer

Extracts classfile annotation information, and adds the annotation info onto the AST.

3. OpenJIT High-level Optimizer Toolkit

The toolkit to construct "compilets", which are modules to specialize the OpenJIT frontend for performing customized compilation and optimizations.

4. Abstract Syntax Tree Package

Provides construction of the AST as well as rewrite utilities.

We first describe the classfile annotation, which is a special feature of OpenJIT, followed by descriptions of the four modules.

\subsection{Classfile Annotation}

Classfile annotation in OpenJIT is additional info or directive added to the classfile to direct OpenJIT to perform classfile-specific (or application-specific, platform-specific) optimization and customization. Here are examples of directives possible with classfile annotations:

- Support for User-defined Optimizers and Specializers

- Support for Memory Models e.g., DSM

- Optimizing Numerical Code

In order to implement the classfile annotation feature, we employ the attribute region of of each method in the classfile. According to the JVM specs, any attributes that the JVM does not recognize are simply ignored; thus, classfiles with OpenJIT annotations can be executed on platforms without OpenJIT, achieving high portability (save for the programs that do not work without OpenJIT). One caveat is that there is no simple way to add extra information in the attribute field of classes themselves, due to the lack of appropriate JIT interface in the 
JVM; thus, one must employ some convention, say, defining a "dummy" null method that is called by the constructor, whose sole purpose is to supply class-wide annotation info that would be cached in the OpenJIT compiler.

In order to create a classfile with annotation information, we either employ an extended version of source-to-bytecode compilers such as javac; for classfiles without source, we could use a tool to add such annotation in an automated way; in fact the tool we are currently testing is a modified version of the OpenJIT frontend system.

\subsection{OpenJIT Bytecode Decompiler Module}

OpenJIT Bytecode Decompiler inputs the bytecode stream from the classfile, and converts it into an augmented AST. The module processes the the bytecode in the following way:

1. Converts the bytecode stream into an internal representation of JVM instruction, and marks the instructions that become the leading instruction of basic blocks.

2. Construct a control flow graph (CFG) with basic block nodes.

3. Construct a dominator tree that corresponds to the CFG.

4. Reconstruct the Java AST by symbolic execution of the instructions within the basic block.

5. Discover the control flow that originated from the short-circuit optimizations of the Java conditional expressions such as \&\& or $\mathrm{I}$ and $(\mathrm{x}$ ? a $* \mathrm{~b})$, and recover the expressions.

6. Reconstruct the Java control structure using the algorithm described in [MOM99].

7. Output the result as an AST, augmented with control-flow and dominator information.

All the above steps except (6) are either simple, or could be done with existing techniques, such as that described in [Ole97]. Step (6), is quite difficult; most previous techniques published so far analyzed the CFG directly, and used pattern matching to extract valid Java control structures [Ole97, TS97]. Instead, we have proposed an algorithm which walks over the dominator tree, and enumerates over every possible patterns of dominance relation, which has a corresponding Java control structure. Compared to existing techniques such as Krakatoa[TS97], our method was shown to be faster, and more robust to code obfuscation. Some preliminary details can be found in [MOM99].

\subsection{OpenJIT Class Annotation Analyzer Module}

The OpenJIT Class Annotation Analyzer module extracts the class annotation from a classfile, and adds the annotation info to the AST. The added annotations are typically compilets that modify the compiler more concretely, it processes the annotation in the following way:

1. First, it access the attribute region of the method. This is done by parsing the method block region extracted from the JVM.

2. We process this byte array assuming that the annotation object has been serialized with writeObject (), constructing an annotation object.

3. we attach the annotation object to the AST as annotation information.

Because what kind of information is to be embodied in the classfile annotation differs according to its usage, the OpenJIT_Annotation is actually an abstract class, and the user is to subclass a concrete annotation class. The abstract superclass embodies the identifier of the annotation, and the AST node where it is to be attached. This is similar in principle to SUIF, except that the annotation must be extracted from the classfile instead of being given a priori by the user. 


\subsection{OpenJIT High-level Optimizer Toolkit}

OpenJIT High-level Optimizer Toolkit is used to construct OpenJIT compilets, that are a set of classes that customizes the compiler. The toolkit provides means of utilizing the augmented AST for implementing traditional compiler optimizations, and is largely composed of the following three submodules: ${ }^{3}$

\section{Flowgraph Constructor}

Flowgraph Constructor creates various (flow) graphs from the augmented AST, such as dataflow graph, FUD chains, control dependence graph, etc. The Flowgraph class is an abstract class, and Factory Method pattern is employed to construct user-defined flowgraphs.

2. Flowgraph Analyzer

The Flowgraph Analyzer performs general computation over the flowgraph, i.e., dataflow equation solving, handling merges, fix point calculation, etc. We employ the Command Pattern to subclass the Analyzer class for each algorithm, and each subclass triggers its own algorithm with the execute() method. The user can subclass the Analyzer class to add his own flowgraph algorithm.

3. Program Transformer

The Program Transformer employs declarative pattern matching and rewrite rules to transform the augmented AST. One registers the rule using the following API:

- register_pattern(Expression src, Expression dst)

- register_pattern (Statement src, Statement dst)

Registers the transformation rule that transforms the src pattern to the dst pattern. The pattern can be constructed using the Abstract Syntax Tree Package described next.

- substitution(Expression root)

- substitution(Statement root)

Searches the subtree with the designated root node depth-first, and if a match is found with the registered patterns, we perform the transformation.

Initial use of the current pattern matching technique proved to be somewhat too low-level; in particular, generation and registration of the transformation rule is still cumbersome. The next version of OpenJIT will have APIs to generate patterns and transformation rules from higher-level specifications, in particular for well-known program transformations (such as code motion, loop transformation, etc.)

\subsection{Abstract Syntax Tree Package}

The Abstract Syntax Tree Package is a utility package called from other parts of the OpenJIT frontend to implement low-level construction of the augmented AST, patterns for transformation rules, etc. The AST essentially implements the entire syntactic entities of the Java programming language. Each node of the AST corresponds to the expression or a statement in Java. The class hierarchy for the package is organized with appropriate subclassing of over 100 classes: (Fig. 3). We show typical Expression and Statement classes in Fig. 4 and Fig. 5, respectively.

A typical Expression subclass for a binary operator (MultiplyExpression in the example) consists of the operator ID, left-hand and right-hand expressions, and reference to an

\footnotetext{
${ }^{3}$ In the current version, compilets are not downloadable; this is primarily due to the fact OpenJIT itself is not yet entirely downloadable due to a few restrictions in the JVM. We are currently working to circumvent the restrictions, and a prototype is almost working. Meanwhile, the Toolkit itself is available, and a custom version of OpenJIT can be created with "static" compilets using standard inheritance.
} 


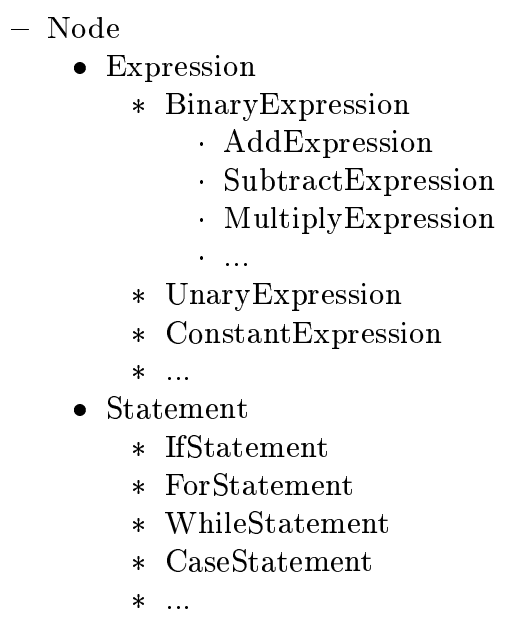

Fig. 3. Class Hierarchy of The Abstract Syntax Tree Package

annotation object. The code() method either generates the low-level IL for the backend, or a Java bytecode stream. The code() method walks over the left- and right-hand expressions in a recursive manner, generating code. When a node has non-null reference to an annotation object, it calls the execute() method of the annotation, enabling customized transformations and compilations to occur.

As a typical Statement subclass, IfStatement recursively generates code for the conditional in a similar manner to Expressions.

As such, the current OpenJIT is structured in a similar manner to OpenC $++[$ Chi95], in that syntactic entities are recursively compiled. The difference is that we provide annotation objects that abstracts out the necessary hook to the particular syntax node, in addition to customization of the syntax node themselves. Thus, it is possible to perform similar reflective extensions as OpenC ++ in an encapsulated way.

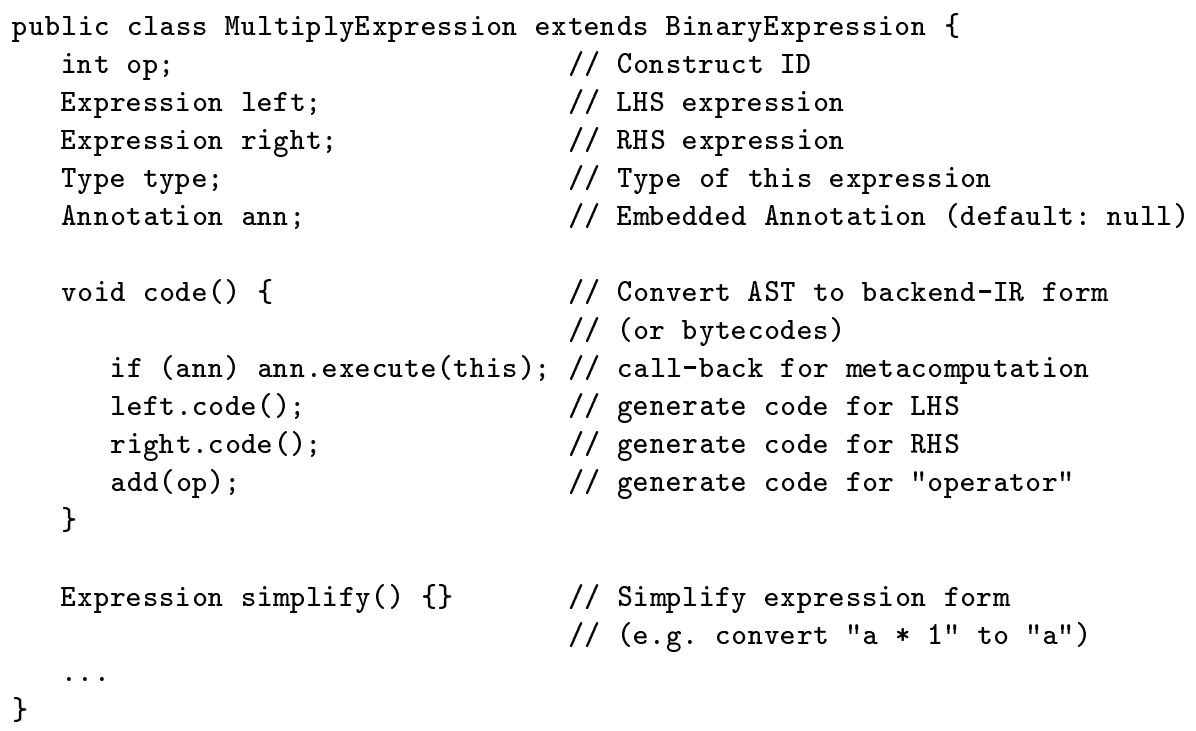

Fig. 4. An Expression Class for A Typical Binary Expression (Multiply) 


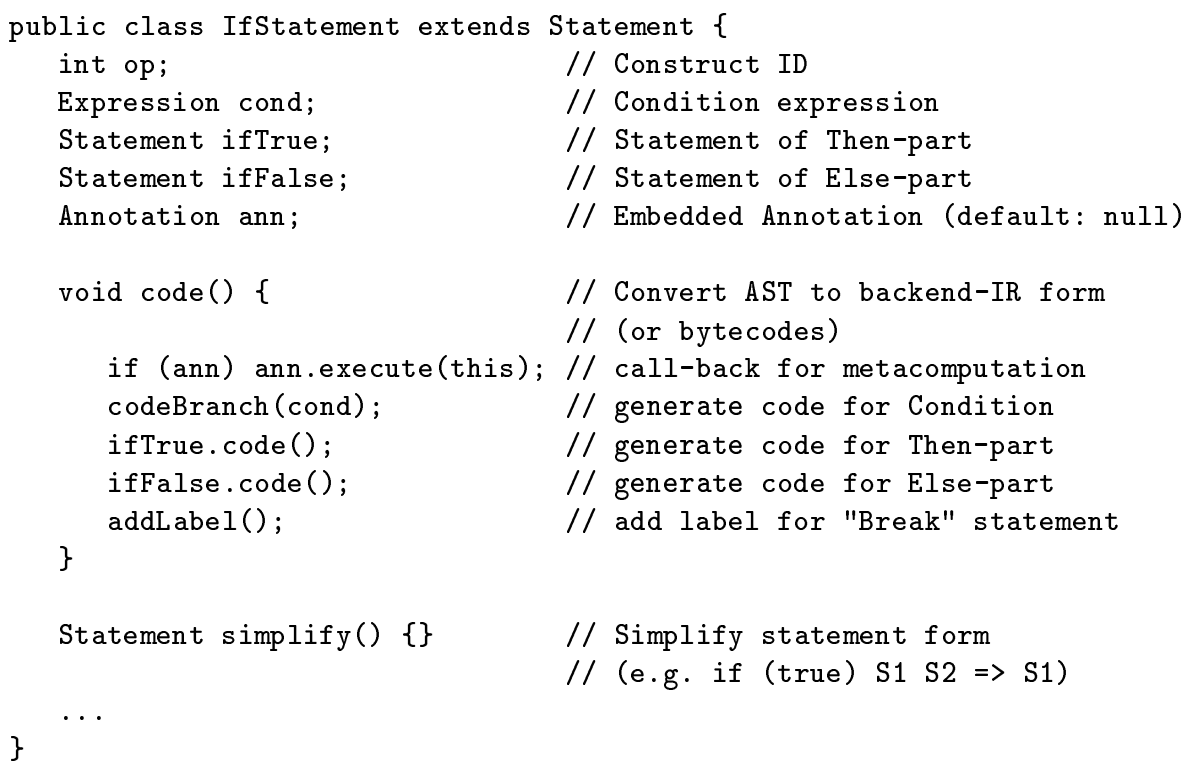

Fig. 5. A Example Statement Class for the "If" Statement

\section{OpenJIT-Backend and its Technical Issues}

\subsection{Overview of the OpenJIT backend system}

As a JIT compiler, the high-level overview of the workings of OpenJIT backend is standard. The heart of the low-level IL translator is the parseBytecode () method of the ParseBytecode class, which parses the bytecode and produces an IL stream. The IL we defined is basically an RISC-based, 3-operand instruction set, but is tailored for high affinity with direct translation of Java instructions into IL instruction set with stack manipulations for later optimizations. There are $36 \mathrm{IL}$ instructions, to which each bytecode is translated into possibly a sequence of these instructions. Some complex instructions are translated into calls into run-time routines. We note that the IL translator is only executed when the OpenJIT backend is used in a standalone fashion; when used in conjunction with the frontend, the frontend directly emits IL code of the backend.

Then, RTL converter translates the stack-based IL code to register based RTL code. The same IL is used, but the code is restructured to be register-based rather than encoded stack operations. Here, a dataflow analyzer is then run to determine the type and the offset of the stack operands. We assume that there are infinite number of registers in this process. In practice, we have found that 24-32 registers are sufficient for executing large Java code without spills when no aggressive optimizations are performed[SK96]. Then, the peephole optimizer would eliminate redundant instructions from the RTL instruction stream.

Finally, the native code generator would generate the target code of the CPU. It first converts IL restricting the number of registers, inserting appropriate spill code. Then the IL sequence is translated into native code sequence, and ISA-specific peephole optimizations are performed. Currently, OpenJIT supports the SPARC and x 86 processors as the target, but could be easily ported to other machines ${ }^{4}$. The generated native code will be then invoked by the Java VM, upon which the OpenJIT runtime module will be called in a supplemental way, mostly to handle Java-level exceptions.

${ }^{4}$ Our experience has been that it has not been too difficult to port from SPARC to $\mathrm{x} 86$, save for its slight peculiarities and small number of registers, due in part being able to program in Java. We expect that porting amongst RISC processors to be quite easy. 


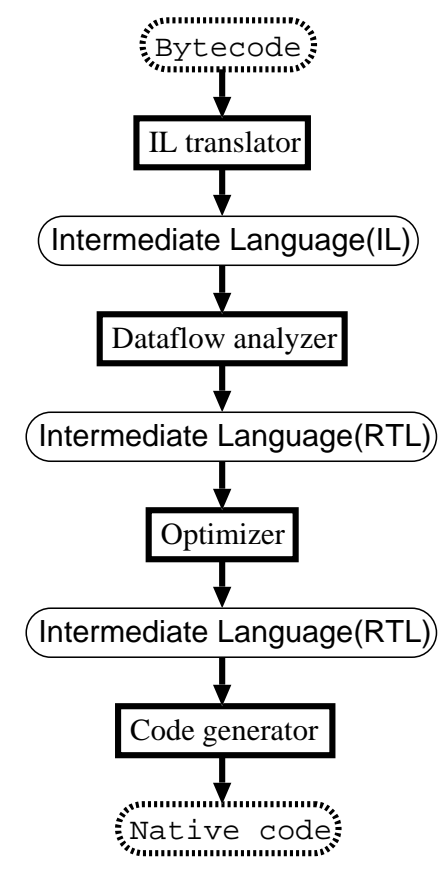

Fig. 6. Overview of the OpenJIT Backend System

The architectural outline of the OpenJIT backend is illustrated in Figure 6. Further details of the backend system can be found in [Shi98].

\subsection{Technical challenges in a Reflective Java JIT compiler}

As most of OpenJIT is written in Java, the bytecode of OpenJIT will be initially interpreted by the JVM, and gradually become compiled for faster, optimized execution. Although this allows the JIT compiler itself to adapt to the particular execution environment the JIT optimizes for, it could possibly give rise to the following set of problems:

1. Invoking the Java-based JIT compiler from within the JVM As the JIT compiler is invoked in the midst of a call chain of the base Java program. There must be a smooth way to massaging the JVM into invoking a JIT compiler in Java in a separate context.

2. Recursive Compilation: The current OpenJIT is designed to be entirely bootstrapped in "cold" mode, i.e., no parts of the JIT compiler are precompiled. Thus, as is with any reflective system, there must be some mechanism to stop the infinite recursive process, and "bottom out". This is a little more subtle than conventional compiler bootstrapping, as compilation occurs at runtime coexisting with compilation of applications; furthermore, the mechanism must be safe w.r.t. Java multi-threading, i.e., no deadlocks should occur.

3. Speed and Memory Efficiency of the JIT compiler: a JIT compiler is beneficial only if the combined (compilation time + execution time) is smaller than the interpretation time under JVM. In more practical terms, OpenJIT must compete with traditional C-based JIT compilers for performance. Here, because of the interpretation and possible slowness of JIT execution even if itself were JIT compiled due to quality of generated native code, it is not clear if such goals could be satisfied. Moreover, memory efficiency is of primary concern, especially for embedded systems. In this regard, there is a particular issue not present in C-based JIT compilers. 


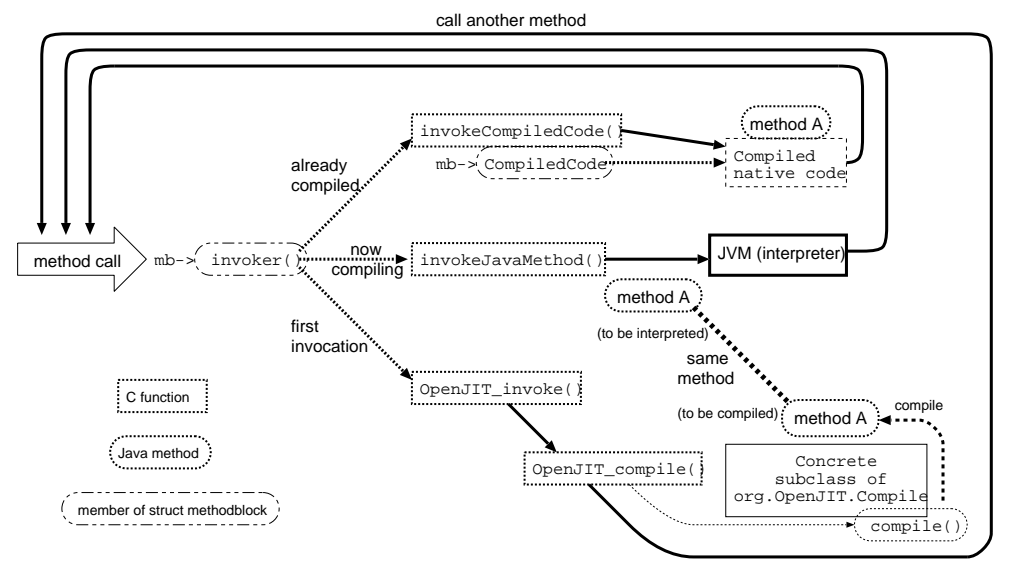

Fig. 7. Invoking OpenJIT

4. Lack of appropriate API for Java-written JIT compilers in standard JVM: A JIT compiler must be able to introspect and modify various data structures within the JVM. Unfortunately, JVM does not have any APIs for that purpose, primarily because it is likely that JIT compilers were assumed to be written with a low-level language such as C. For this purpose, there must be appropriate Java-level APIs which must be reasonably portable for JVM introspection in OpenJIT.

We brevety, we only cover the most salient technical features here: for complete technical details readers are referred to [SM99].

Invoking the Java-based JIT compiler from within the JVM In a "classic" JVM, for each method, both JIT compilation and transfer of control to the native method happens at the point of the subject method invocation. The JVM interpreter loop is structured as follows. When a method is invoked, the invoker function of the methodblock structure (a structure internal to the JVM which embodies various info pertaining to a particular method) $\mathrm{mb}$ is called. Under interpretive execution, this in turn calls the JVM to generate a new Java stack frame. The first argument of invoker () function o is the class object for static method calls, and the invoked object on normal method calls. The second argument mb is a pointer to the methodblock structure, etc.

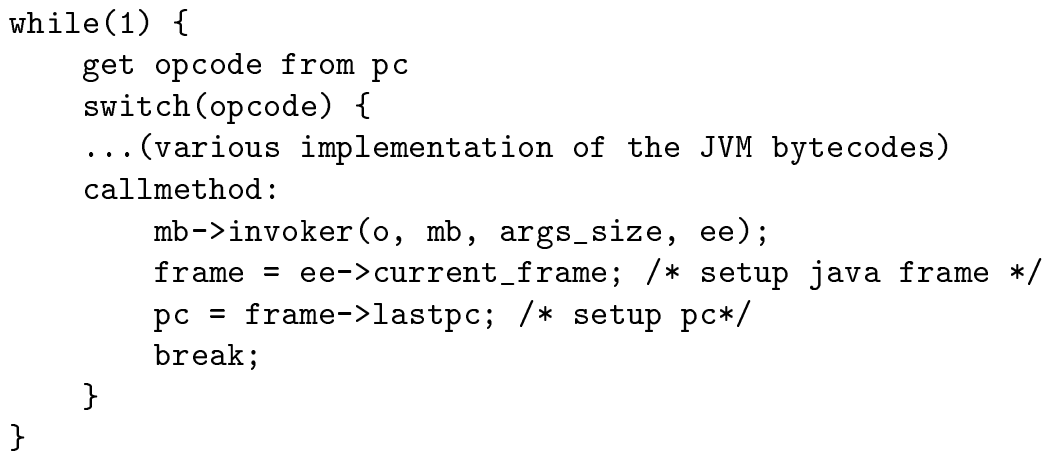

We substitute the value of the invoker in methodblock structure of every method to OpenJIT_invoke when a class is loaded. The OpenJIT_invoke function is defined as follows in $\mathrm{C}$ : 
bool_t OpenJIT_invoke(JHandle *o, struct methodblock *mb, int args_size, ExecEnv *ee);

This function in turns calls the OpenJIT_compile() in the $\mathrm{C}$ runtime to dynamically compile the method. Thereafter, the control is transferred to mb->invoker, transferring control to the just compiled method. The called OpenJIT_compile performs the following functions:

Mutual exclusion to prevent simultaneous compilation of the same method We must prevent multiple threads from compiling the same method at the same time with proper mutual execution using a compile lock. We reserve a bit in the methodblock structure as a lock bit.

Setup of invoker and CompiledCode fields in the methodblock structure When a method is invoked, and subject to compilation, we reset the invoker and other fields in the methodblock so that any subsequent invocation of the method will have the method run by the interpreter during compilation. This allows natural handling of recursive selfcompilation of OpenJIT compiler classes.

Invocation of the body of the JIT compiler The Java method to invoke the compiler is then upcalled. An instance of a new JIT compiler in Java (to be more specific, its upcall entry class) is allocated and initialized for each JIT compiler invocation. Then, the compile () method of the instantiated entry class is up with do_execute_java_method_vararg(). Note that the current call context is preserved in the stack; that is to say, the same thread is utilized to make the upcall.

Postprocessing of JIT compilation After compilation, control returns to the $\mathrm{C}$ runtime. At this point, most of the compiler becomes garbage, except for the persistent information that must be maintained across method compilations. This is to facilitate dynamic change in the compiler with compilets, and also to preserve space, directly exploiting the memory management feature of Java. If the compilation is successful, we set the invoker field of the methodblock structure to the compiled native code. When compilation fails: The methodblock field values are restored to their original values. ${ }^{5}$

In this manner, the JIT compiler in Java is smoothly invoked on the same execution thread. In practice it is much more complicated, however, due to possibility of exceptions, JIT compilation occurring even on calls from native methods, advanced features such as backpatching, inlining, and adaptive compilation. Some of the issues are further discussed below, while for the rest refer to [SM99].

Recursive Compilation Recursive compilation is handled at the $\mathrm{C}$ runtime level of OpenJIT with simple locking mechanism, as we see in the following simplified code fragment (in practice, it would include more code such as support for adaptive compilation):

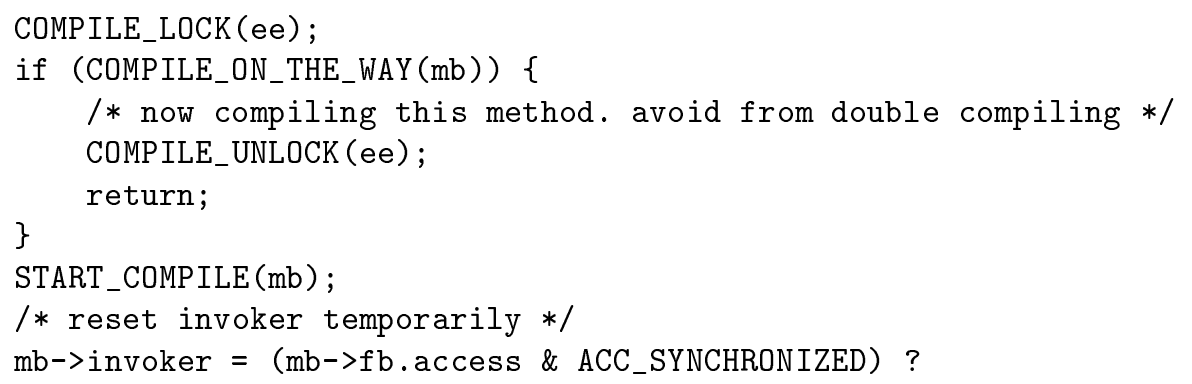

${ }^{5}$ In practice, the invoker field is not directly substituted for the compiled native method, but rather we invoke a native code stub, depending on the type of the return argument. This is done to handle exceptions, java reflection, calls between native and interpreted code, etc. 


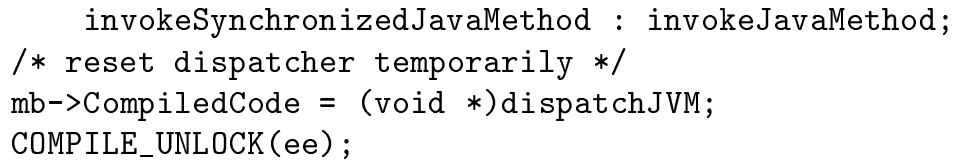

This is essentially where the compilation "bottoms out"; once the method starts to be compiled, a lock is set, and further execution of the method will be interpreted. In fact, in Java we actually obtain this behavior for free, as mutual exclusion of multi-threaded compilation has to be dealt with in any case, defaulting to interpretation.

However, in the case of recursive compilation, there are some issues which do not exist for C-based JIT compilers:

- Possibility of Deadlocks - We must be assured that, as long as JIT compiler obeys the locking protocol, recursive multi-threaded compilation does not cause any deadlocking situations. This is proven by showing that cyclic resource dependencies will not exist between the multi-threaded compilations. Let the dependencies between the methods be denoted $m_{1}^{c} \longrightarrow m_{2}^{c}$, where for execution of compiled method $m_{1}^{c}$ we need to execute a compiled method $m_{2}^{c}$. We further distinguish compiled and interpreted execution of methods with $m^{c}$ and $m^{i}$, respectively. Then, starting from the entry method as a node, graph of dependency relations will clearly form a tree for single-threaded case. For multithreaded case, however, it must be shown that arbitrary interleavings of the tree via possible self compilation will only create DAGs. Informally this simply holds because all $m_{i}$ 's will not be dependent on any other nodes, and thus the cycle will have to be formed amongst $m_{c}$ 's, which is not possible. The formal proof will be a subject of our future paper.

We also note that, in practice, deadlocks could and does occur not only between the JIT compiler and the JVM. One nasty bug which took a month to discover was in fact such a deadlock bug. As it turns out, the "classic" JVM locks the constant pool for a class when its finalizer is run. This could happen just when OpenJIT tries to compile the finalizer method, resulting in a deadlock.

- Speed and Memory Performance Problems - Aside from the JIT compiler merely working, we must show that the JIT compiler in Java could be time and memory efficient. The issue could be subdivided into cases where the OpenJIT is compiling (1) application methods, and (2) OpenJIT methods. The former is simply shown by extensive analysis of standard benchmarks in Section 5, where it is shown that OpenJIT achieves good time and memory performance and despite being constrained by the limitations of the "classic" VM, such as handle-based memory systems implementation, non-strict and non-compacting GC, slow monitor locking, etc. The latter is much more subtle: because of recursive compilation, two undesirable phenomena occur. (A) compilation of a single application bytecode will set off a chain of recursive compilations, due to the dependency just discussed. This has the effect of accumulating compiling contexts of almost the entire OpenJIT system, putting excessive pressure on the memory system. (B) We could prevent the situation by employing adaptive compilation and defaulting back to interpretation earlier, but this will have the effect of slowing down the bootstrap time, as long as possibly having some residual effect on application compilation due to some OpenJIT compiler methods still being interpreted.

(A) and (B) are strongly interrelated; in the worst case, we will be trading speed, especially the bootup time, for space. On the other hand, one could argue that little penalty is incurred by adaptive means, not because of the typical execution frequency argument, but rather, that because of recursive compilation, much of the OpenJIT system could be compiled under interpretation in the first place. We perform extensive performance analysis to investigate this issue in Section 5. 
Lack of appropriate API for Java-written JIT compilers in standard JVM None of the current Java VMs, including the "classic" VM for which OpenJIT is implemented, have sufficient APIs for implementing a JIT compiler in Java. In particular, JVM basically only provides APIs to invoke a C-based JIT compiler, but does not provide sufficient APIs for generalized introspection or intersession features. Note that we cannot employ the Java reflection API either, for it abstracts out the information required by the JIT compiler.

Instead, we define a set of native methods as a part of the OpenJIT runtime. The Compile class declares the following native methods, which are defined in api.c of the distribution. There are 17 methods in all, which can be categorized as follows:

\section{- Constant pool introspection methods}

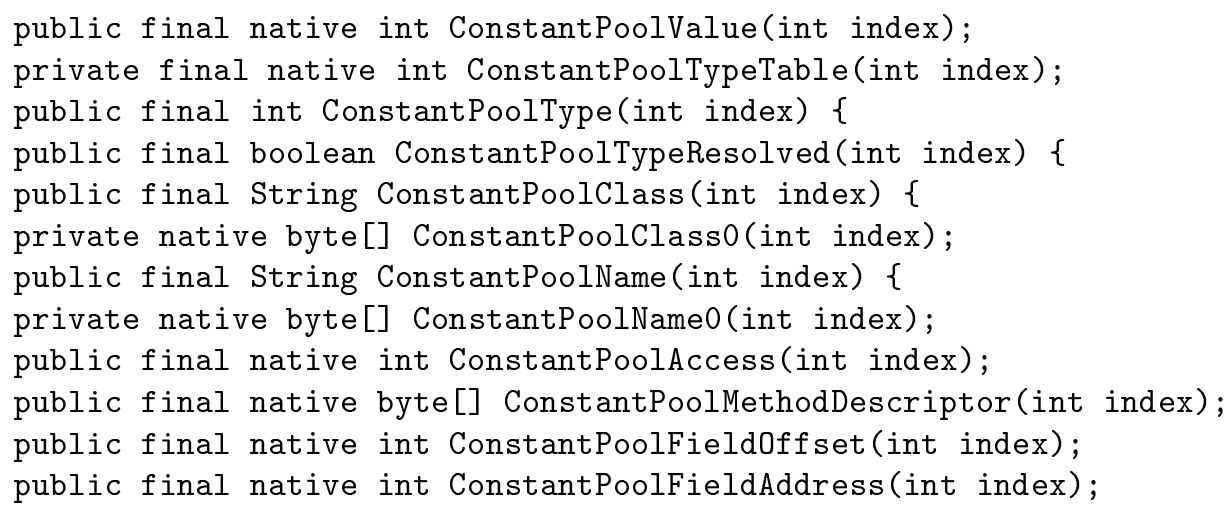

\section{- Native method allocation and reflection}

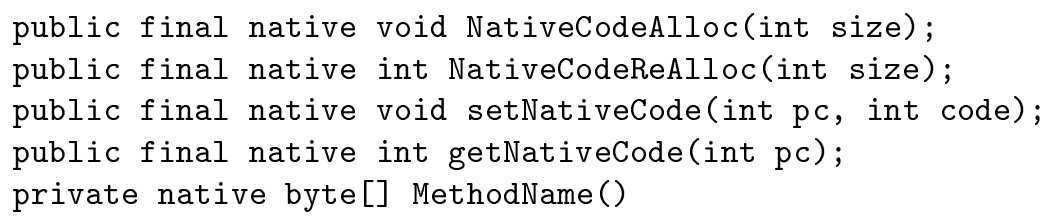

- Class resolution methods (used for inlining)

public final native void initParser(int caller_cp, int index); public final native void resolveClass(int caller_cp, int index)

As one can see, majority of the methods are such that either introspective or intercessive operations being performed on the JVM.

The current API is sufficient, but admittedly too low level of abstraction, in that it exposes too much of the underlying VM design; indeed, our goal is to allow JITs to be a customizable and portable hook to the Java system, and thus, have OpenJIT be portable across different kinds of VMs. For this purpose, in the next version of OpenJIT, we plan to design a substantially higher-level API, abstracting out the requirements of the different VMs. The implementation of the API for "classic" VM will sit on the current APIs, but other VMs will have different implementations of native methods.

Another issue is the safety of the API. In the current implementation, the OpenJIT native method APIs are accessible to all the classes, including the application classes. It is easy to restrict the access to just the compiler classes (those with path org.OpenJIT.), but this will preclude user-defined compilets. Some form of security/safety measures with scope control, such as restricting access only to signed classfiles, might be necessary. We are currently investigating this possibility to utilize the security API in JDK 1.2. 


\section{Performance Analysis of OpenJIT}

We now analyze the behavior of OpenJIT with detailed benchmarks. As mentioned earlier, our concern is both execution speed and memory usage. The former is obvious, as the execution overhead of the JIT compiler itself as well as quality of generated code will have to match that of conventional JIT compilers. Memory usage is also important, especially in areas such as embedded computing, one of major Java targets.

All the OpenJIT objects, except for the small C runtime system, coexists in the heap with the target application. The necessary working space includes that of various intermediate structures of compiler metaobjects that the OpenJIT builds, including various flowgraphs, intermediate code, etc., and persistent data, such as the resulting native code. Standard Cbased JIT compilers will have to allocate such structures outside the Java heap; thus, memory usage is fragmented, and efficient memory management of the underlying JVM is not utilized. For OpenJIT, since both the application and compiler metaobjects will coexist in the heap, it might seem that we would obtain the most efficient usage of heap space.

On the other hand, the use of Java objects, along with automated garbage collection, could be less memory efficient than C-based JITs. Moreover as mentioned in Section 4, there could be a chain of recursive compilations which will accumulate multiple compilation contexts, using up memory. It is not clear what kind of adaptive compilation techniques could be effective in decreasing the accumulation, while not resulting in substantial execution penalty.

\subsection{Benchmarking Environment}

As an Evaluation Environment, we employed the following platform, and pitted OpenJIT against Sun's original JIT compiler (sunwjit) on JDK 1.2.2 (ClassicVM).

- Sun Ultra60 (UltraSparc II $300 \mathrm{MHz} \times 2,256 \mathrm{MB}$ )

- Solaris 2.6-J

- JDK 1.2.2 (ClassicVM)

We took six programs from the SPECjvm98 benchmark, as well as the simple "Hello World" benchmark. The six__201_compress (file compression),_202_jess (expert system), 209_db (DBMS simulator),_213_javac (JDK 1.0.2 compiler), and _227_mtrt (multi-threaded raytracer), and _228_jack (parser generator) — have been chosen as they are relatively compute intensive, do not involve mere simple method call loops, and not reliant on runtime native calls such as networks, graphics, etc. "Hello World" benchmark superficially only makes a call to System.out.println(), but actually it will have executed almost the entire OpenJIT system, the Java packages that OpenJIT employs, as well as the constructors of system classes. This allows us to observe the bootstrap overhead of the OpenJIT system.

In order to obtain the precise profile information for memory allocation, we employed the JVMPI (Java Virtual Machine Profiler Interface) of JDK 1.2.2. Additionally, we extended OpenJIT to output its own profile information. This is because it is difficult to determine with JVMPI whether the allocated compiler metaobject is being used to compile application methods, or used for recursive compilation, because JVMPI merely reports both to be of the same class (say, merely as instances of ILnode, etc.). By combining JVMPI and OpenJIT profile information, we obtain precise information of how much space the live OpenJIT compiler metaobjects occupy, how much native code is being generated, how much of the native code is that of OpenJIT, along the execution timeline. Also, how much classfiles are being loaded, how many methods are being compiled, and what is the percentage of the OpenJIT classes, can be profiled as well.

Such profiling is done in real-time, in contrast to the simulation based profiling of SpecJVM memory behavior in [DH99]. Such an approach is difficult to apply for our purpose, as JIT 
Table 1. Code size of OpenJIT and C runtimes

\begin{tabular}{|l|r|r|r|r|}
\hline & classes (files) & methods & \# lines & classfile (stripped binary) bytes \\
\hline \hline Frontend & 243 & 1,439 & 24,148 & 629,062 \\
Backend (sparc) & 23 & 182 & 7,560 & 118,592 \\
Backend (x86) & 21 & 182 & 8,085 & 118,125 \\
C runtime(sparc) & 3 & & 3,565 & 42,556 \\
C runtime(x86) & 3 & & 3,752 & 28,928 \\
sunwjit (sparc) & & & & 234,112 \\
sunwjit (x86) & & & & 146,508 \\
\hline
\end{tabular}

compilation is being directly involved, resulting in code not directly profilable with JVM simulation. Our compiler-assisted profiling allows us to obtain almost as precise an information as that of [DH99] at a fraction of time. Nevertheless, the profile information generated is quite large, reaching several hundred megabytes for each SpecJVM run.

\subsection{Benchmarking Contents}

The Size of OpenJIT We first show the code size of OpenJIT compared to sunwjit. As we can see, the frontend is approximately 3 times the size of backend in terms of number of lines, and factor of approximately 8-10 larger in terms of number of classes and methods. This is because the frontend contains numerous small classes representing syntactic entities of Java, whereas the backend has much larger method size, and the backend IL does not assign a class for each instruction. We also see that the combined size of OpenJIT backend and C runtime is smaller than sunwjit, but when it is self-compiled, the x 86 version could get larger. Thus, this raises an interesting issue of what happens if we run the compiler always interpreted in embedded situations; in the subsequent benchmark, we will also investigate this possibility.

Baseline Performance We next observe the baseline execution time and memory usage characteristics of OpenJIT. We set the heap limit to 32MBytes (as mandated by the SpecJVM98 benchmarks) comparing the execution of JVM interpreter, sunwjit, OpenJIT with self compilation, and OpenJIT without self compilation. Table 2 shows for each execution, how many classes are loaded and their sizes, how many objects are allocated (parenthesis indicates how many OpenJIT compiler metaobjects), how much memory size are allocated (and that of OpenJIT compiler metaobjects), wallclock execution time, and number of GCs.

Figure 8 additionally show consumed overall heap space, live OpenJIT object heap space, along the time axis. This shows the process of compiler bootstrapping. The compilation in OpenJIT was set to be most aggressive i.e., all the methods are JIT compiled on their first invocations, and the entire frontend had been turned off, and are not loaded.

The Hello benchmark exemplifies the overhead of bootstrapping openjit and openjit-int; compared to sunwjit, we see approximately 2.8 times increase in startup time, indicating that compilation with OpenJIT incurs approximately $\times 3$ overhead over sunwjit. On the other hand, different between openjit and openjit-int is negligibly small; this indicates that overhead of self compilation is almost negligible, but rather, the overhead of system and library classes are substantial (we observe approximately 457 methods compiled, as opposed to 128 methods for OpenJIT).

For the six SPECjvm98 benchmarks, we see that the overhead is well amortized, and OpenJIT is competitive with sunwjit, sometimes superior. The running time of programs range between 56-172 seconds, so the overhead of JIT compilation is well amortized, even for openjit-int, given the relative expense of OpenJIT compilation over sunwjit. Moreover, since method-specific openjit compiler metaobjects are mostly thrown away on each compilation, in principle we do not occupy memory compared to sunwjit (Fig. 8) In fact, we may be 
Table 2. Baseline Performance

\begin{tabular}{|c|c|c|c|c|c|c|}
\hline Program & JIT & class\# & $\begin{array}{r}\text { alloc obj\# } \\
\text { (openjit) }\end{array}$ & $\begin{array}{r}\text { allocsize[MB] } \\
\text { (openjit) }\end{array}$ & GC\# & time \\
\hline \multirow{4}{*}{ Hello } & interpreter & 167 & $4,890(-)$ & $0.273(-)$ & 0 & 0.380 \\
\hline & sunwjit & 172 & $5,244(-)$ & $0.285(-)$ & 0 & 0.450 \\
\hline & openjit & 185 & $90,600(74,831)$ & $2.906(2.316)$ & 5 & 1.270 \\
\hline & openjit-int & 185 & $37,059(31,149)$ & $1.265(0.941)$ & 2 & 1.280 \\
\hline \multirow{4}{*}{$\begin{array}{c}-201_{-} \\
\text {compress }\end{array}$} & interpreter & 224 & $15,547(-)$ & $110.640(-)$ & 20 & 673.910 \\
\hline & sunwjit & 226 & $9,399(-)$ & $110.266(-)$ & 16 & 89.620 \\
\hline & openjit & 241 & $136,328(107,197)$ & $114.330(3.318)$ & 21 & 72.530 \\
\hline & openjit-int & 241 & $81,910(62,742)$ & $112.662(1.918)$ & 18 & 74.460 \\
\hline \multirow{4}{*}{$\begin{array}{l}-202 \_ \\
\text {jess }\end{array}$} & interpreter & 373 & $7,951,562(-)$ & $221.919(-)$ & 547 & 148.550 \\
\hline & sunwjit & 375 & $7,936,214(-)$ & $221.190(-)$ & 565 & 65.750 \\
\hline & openjit & 390 & $8,103,973(142,626)$ & $226.383(4.402)$ & 528 & 62.530 \\
\hline & openjit-int & 390 & $8,049,403(98,045)$ & $224.710(2.998)$ & 532 & 62.160 \\
\hline \multirow{4}{*}{$\begin{array}{c}209 \\
\mathrm{db}\end{array}$} & interpreter & 218 & $3,218,293(-)$ & $63.249(-)$ & 33 & 307.480 \\
\hline & sunwjit & 220 & $3,213,851(-)$ & $63.095(-)$ & 32 & 142.160 \\
\hline & openjit & 235 & $3,343,820(109,778)$ & $67.104(3.398)$ & 39 & 172.830 \\
\hline & openjit-int & 235 & $3,289,249(65,197)$ & $65.431(1.994)$ & 37 & 182.080 \\
\hline \multirow{4}{*}{$\begin{array}{l}213_{-} \\
\text {javac }\end{array}$} & interpreter & 386 & $5,972,713(-)$ & $147.288(-)$ & 80 & 200.940 \\
\hline & sunwjit & 388 & $5,936,663(-)$ & $145.458(-)$ & 69 & 94.850 \\
\hline & openjit & 403 & $6,181,295(208,562)$ & $154.486(6.478)$ & 77 & 102.960 \\
\hline & openjit-int & 403 & $6,126,571(164,145)$ & $151.531(5.080)$ & 67 & 108.850 \\
\hline \multirow{4}{*}{$\begin{array}{l}-227 . \\
\text { mtrt }\end{array}$} & interpreter & 239 & $6,382,222(-)$ & $84.118(-)$ & 90 & 173.510 \\
\hline & sunwjit & 241 & $6,376,266(-)$ & $83.902(-)$ & 90 & 59.430 \\
\hline & openjit & 256 & $6,524,115(124,549)$ & $88.467(3.855)$ & 96 & 56.640 \\
\hline & openjit-int & 256 & $6,469,545(79,968)$ & $86.794(2.451)$ & 93 & 56.980 \\
\hline \multirow{4}{*}{$\begin{array}{l}228 \\
\text { jack }\end{array}$} & interpreter & 270 & $6,878,777(-)$ & $150.755(-)$ & 451 & 196.330 \\
\hline & sunwjit & 272 & $6,868,951(-)$ & $150.353(-)$ & 465 & 66.669 \\
\hline & openjit & 287 & $7,046,695(152,625)$ & $155.818(4.707)$ & 286 & 66.970 \\
\hline & openjit-int & 287 & $6,992,109(108,001)$ & $154.144(3.302)$ & 276 & 68.010 \\
\hline
\end{tabular}



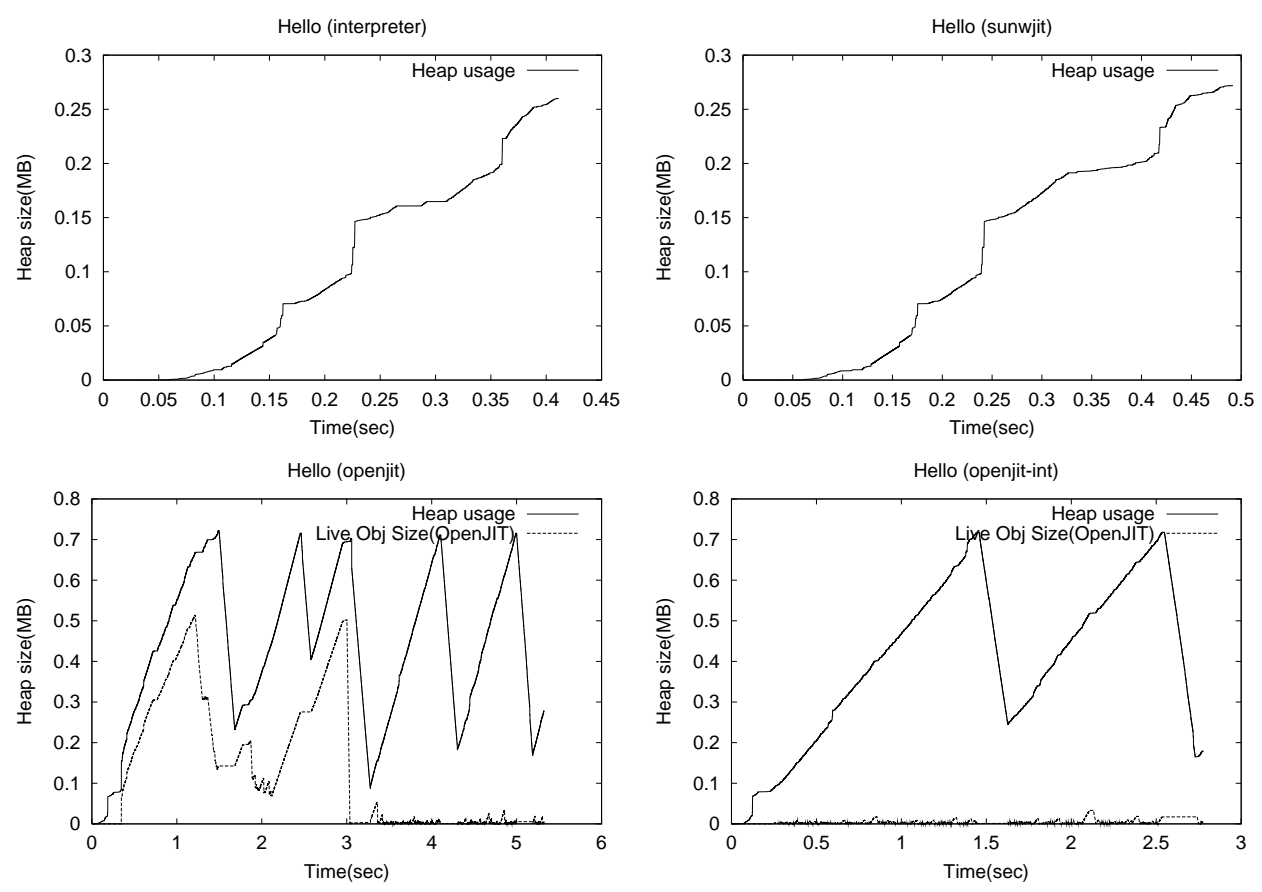

Fig. 8. Timeline behavior of heap usage and live object allocated by OpenJIT (interpreter, sunwjit, openjit, openjit-int)

utilizing memory better due to sharing of the heap space with the application. The runtime comparison of execution times of each program depends on each program. For compress, openjit was $20 \%$ superior, whereas sunwjit was faster by about $18 \%$. Other benchmarks are quite similar in performance. Even small but unnegligible difference in compilation overhead, OpenJIT is likely producing slightly superior code on average.

We do observe some anomalies, however. Firstly, for most cases OpenJIT had increased invocations of GCs due to heap coexistence; but for jack and jess, OpenJIT had less GC invocations, by approx. $40 \%$ and $5 \%$, respectively. This is attributable to unpredictable behavior of conservative GC in the "classic" JVM; it is likely that by chance, the collector happened to mistake scalars for pointers on the stack. Neither really contributes significantly to performance differences. Another anomaly is that, in many cases openjit-int was faster than openjit with self-compilation. This somewhat contradicts our observation that compilation DOES incur some overhead, as difference between interpreted and compiled executions of OpenJIT itself should manifest, but doesn't.

Figure 8 shows the timeline track of the amount of heap usage by the entire program, OpenJIT (openjit) and interpreted OpenJIT (openjit-int), respectively, for the Hello benchmark. Again, we observe that during bootstrapping, openjit and openjit-int require approximately $700 \mathrm{Kbytes}$ of heap space, which is about 2.6 times the heap space as sunwjit and pure interpreter. Since openjit-int does not allocate metaobjects to compile itself, and the amount being consumed to compile methods of other classes are small, we attribute the consumption to the system objects with the libraries being called from OpenJIT, and immediately released.

The Hello benchmark also verifies that there are two phases of execution for OpenJIT. Firstly, there is a bootstrap phase where the entire OpenJIT is aggressively compiled, accumulating multiple compilation contexts in the call chain of the JIT compiler. Thus, the space required is proportional to the critical path in the call chain. Then, it quickly falls off, and 

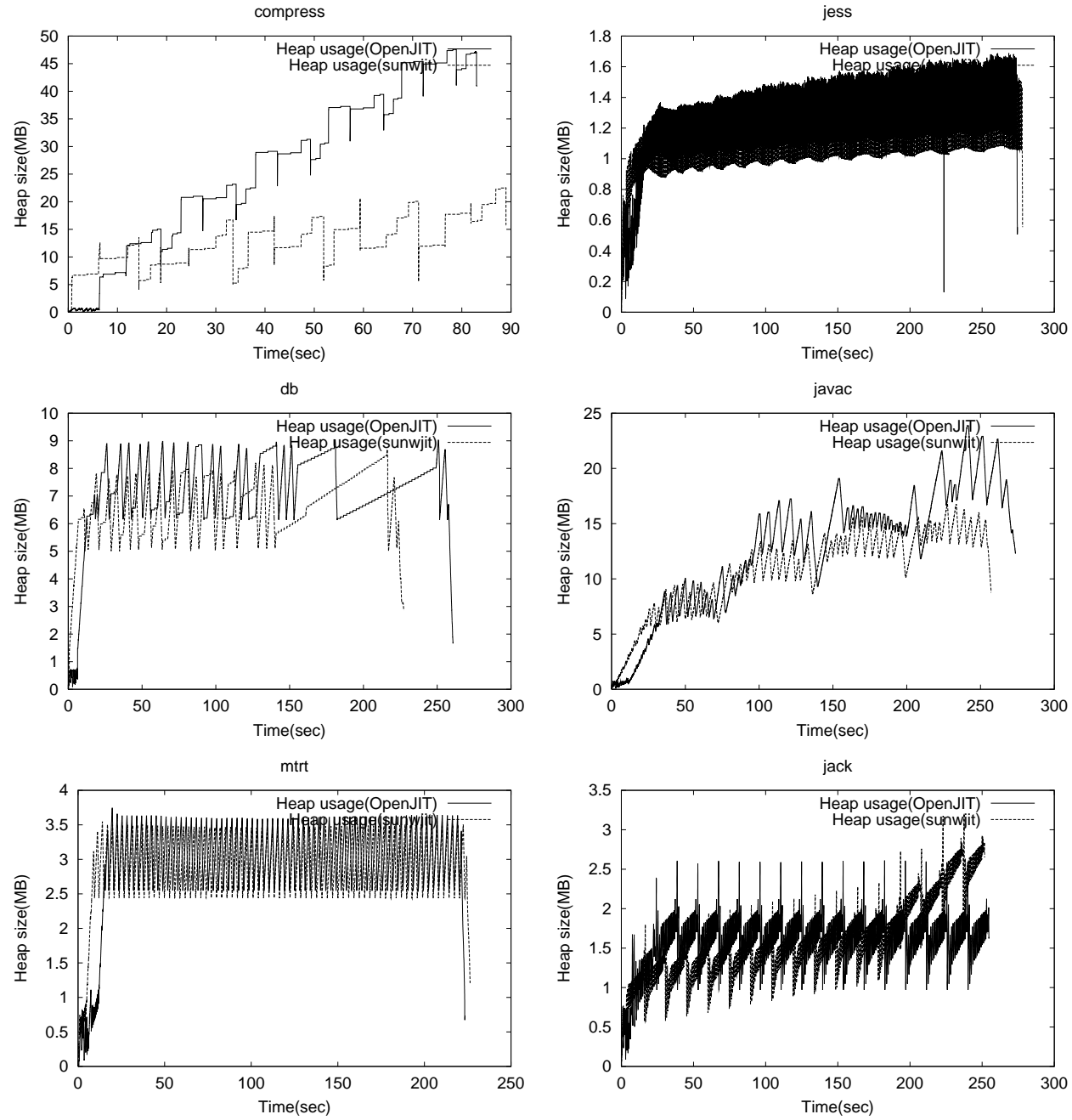

Fig. 9. Timeline behavior of heap usage with SPECjvm98 (sunwjit vs. openjit) 
Table 3. SPECjvm98 results on Linux (x86)

\begin{tabular}{|l|r|r|r|r|}
\hline Benchmark & OpenJIT & interpreter & sunwjit & IBM1.1.8 \\
\hline \hline 200_check & 0.043 & 0.082 & 0.106 & 0.042 \\
\hline _201_compress & 40.828 & 316.687 & 59.338 & 18.236 \\
\hline _202_jess & 29.783 & 90.434 & 48.493 & 13.142 \\
\hline _209_db & 85.881 & 203.289 & 119.228 & 40.259 \\
\hline _213_javac & 56.227 & 120.199 & 70.698 & 30.964 \\
\hline _222_mpegaudio & 40.911 & 263.870 & 41.705 & 11.942 \\
\hline _227_mtrt & 30.101 & 96.156 & 37.337 & 17.014 \\
\hline _228_jack & 28.403 & 107.049 & 49.176 & 10.751 \\
\hline
\end{tabular}

transcends into a stable phase where most parts of OpenJIT have been compiled, and only application methods are being compiled and executed.

No matter how the memory is being used, the amount of additional heap space required for recursively compiling OpenJIT will not be a problem for modern desktop environments, in some situations. A typical desktop applications consumes orders of magnitude more space: for example, our measurements in Figure 9 for javac shows it consumes more than 20 Mbytes $^{6}$. However, for embedded applications, such an overhead might be prohibitive. As discussed earlier, this could be suppressed using less aggressive, adaptive compilation similar to the Self compiler[Hol95], but it is not clear what strategy will achieve good suppression while not sacrificing performance. In the next section we consider several adaptive strategies for suppression.

We have also taken some benchmarks on the x86 version of OpenJIT, and compared it against IBM's JDK 1.1.8 JIT compiler, which is reputed to be the fastest JIT compiler for $\mathrm{x} 86$, in neck to neck with Sun's Hotspot. Table 3 shows the results: we see that, for most benchmarks, OpenJIT x86 is superior to sunwjit, and runs about half the speed of IBMs JIT, despite being constrained by the "classic" JVM.

\subsection{Adaptive OpenJIT Compilation Strategies}

There are several criteria in the design space for adaptive compilation in OpenJIT for memory suppression of the bootstrapping phase.

1. Alteration of JIT compilation frequency-

The most aggressive strategy will compile each method on its first invocation. We reduce the frequency of compilation using the following strategies, with $p$ as a parameter $(p=$ $2,4,8,16)$

- JIT compile on $p$ th invocation, deferring to interpretation for the first $p-1$ invocations (constant delay).

- Assign each method a random number between [0,p-1], and compile when the number of invocation reaches that number (random delay).

- Compile with probability $1 / p$ on each invocation (probabilistic). reduced probability increased the execution time by

2. Restriction of methods subject to adaptation-

We could delay compilation for all the methods, or alternatively, only those of the OpenJIT compiler metaclasses. The former obviously will likely consume less space, but the former may be sufficient and/or desirable, as it will not slow down the application itself. We verify this by comparing altering compilation frequency changes to all classes, versus only altering the frequency of OpenJIT method. For the latter, all other methods are compiled on first invocation except for class initializers, which are interpreted.

${ }^{6}$ [DH99] reports that with exact GC, the actual usage is approximately 6 MBytes. The difference is likely to be an artifact of conservative GC, our close examination has shown. 
Table 4. Alteration of JIT compilation frequency for all methods

\begin{tabular}{|c|c|r|r|r|r|r|}
\hline \multirow{2}{*}{ criteria } & \multicolumn{5}{|c|}{ Hello } \\
\cline { 2 - 7 } & param & $\begin{array}{r}\text { method\# } \\
\text { (openjit) }\end{array}$ & $\begin{array}{r}\text { alloc obj\# } \\
\text { (openjit) }\end{array}$ & $\begin{array}{r}\text { alloc size [MB] } \\
\text { (openjit) }\end{array}$ & GC\# & time \\
\hline \hline always & - & $457(128)$ & $90,594(74,831)$ & $2.905(2.316)$ & 5 & 1.270 \\
\hline constant & 2 & $225(126)$ & $64,827(52,194)$ & $2.117(1.629)$ & 3 & 1.190 \\
delay & 4 & $180(116)$ & $57,568(46,133)$ & $1.898(1.445)$ & 3 & 1.280 \\
& 8 & $161(114)$ & $55,743(44,470)$ & $1.843(1.395)$ & 4 & 1.250 \\
& 16 & $151(113)$ & $54,069(43,064)$ & $1.793(1.352)$ & 4 & 1.810 \\
\hline random & 2 & $412(127)$ & $84,739(69,746)$ & $2.729(2.162)$ & 5 & 1.270 \\
delay & 4 & $301(123)$ & $72,686(59,272)$ & $2.357(1.843)$ & 4 & 1.190 \\
& 8 & $231(120)$ & $62,628(50,589)$ & $2.050(1.578)$ & 4 & 1.240 \\
& 16 & $196(115)$ & $61,923(49,790)$ & $2.031(1.558)$ & 3 & 1.350 \\
\hline probability & 2 & $170(115)$ & $56,383(45,041)$ & $1.862(1.412)$ & 4 & 1.280 \\
& 4 & $190(115)$ & $58,493(46,877)$ & $1.924(1.466)$ & 4 & 1.900 \\
& 8 & $149(115)$ & $53,710(42,735)$ & $1.780(1.341)$ & 3 & 2.010 \\
& 16 & $112(99)$ & $27,066(21,399)$ & $0.955(0.664)$ & 1 & 0.920 \\
\hline
\end{tabular}

\section{Restriction of number of simultaneous compilations-}

We put global restriction on how many compilations can occur simultaneously. This can be done safely without causing deadlocks. Attempt to compile exceeding this limit will default back to the interpreter. $(L=1,8)$. Note that, although simultaneous compilation could occur for application methods under multi-threading, this primarily restricts the simultaneous occurrence of deep recursive compilation chains on bootstrapping.

4. Restricting compilation of org.OpenJIT.ParseBytecode.parseBytecode()

This is a special case, as preliminary benchmarks indicated that parseBytecode(), is quite large for a single method, (1576 lines of source code, $6861 \mathrm{JVM}$ bytecodes), and thus single compilation of this method creates a large structure in the heap space once it is subject to compilation, irrespective of the strategies used. In order to eliminate the effect, we test cases where compilation of parseBytecode () is restricted. In the next version of OpenJIT we plan to factor the method into smaller pieces.

According to the Hello benchmark, in when adaptaion is applied to all the methods, combinations of other schemes effectively yielded reduction in the number and size of objects that are allocated during bootstrapping, without significant increase in bootstrap time. On the other hand, restricting compilation of OpenJIT method only did not yield significant results, except for the case when the entire OpenJIT was interpreted, or when parseBytecode() was restricted, again, without significant loss of performance.

The table only shows the total memory allocated. In order to characterize the peak memory behavior, we present the timeline behavior in Figure 10. Here, for each scheme, the parameter with lowest peak is presented. We observe that, (1) probabilistically lowering the frequency helps reduce the peak usage, and (2) parseBytecode dominates the peak. We are currently conducting futher analysis, but it is conclusive that naive frequency adjustment does not help to reduce the peak; rather, the best strategy seems to be to estimate the heap usage based on bytecode length, and supressing compilation once a prescribed limit is exceeded. We should be able to report on this in the camera-ready version of the paper. 
Table 5. Alteration of JIT compilation frequency for OpenJIT methods only

\begin{tabular}{|c|c|r|r|r|r|r|}
\hline \multirow{2}{*}{ criteria } & \multicolumn{6}{|c|}{ Hello } \\
\cline { 2 - 7 } & param & $\begin{array}{r}\text { method\# } \\
\text { (openjit) }\end{array}$ & $\begin{array}{r}\text { alloc obj\# } \\
\text { (openjit) }\end{array}$ & $\begin{array}{r}\text { alloc size }[\mathrm{MB}] \\
\text { (openjit) }\end{array}$ & GC\# & time \\
\hline \hline always & - & $457(128)$ & $90,594(74,831)$ & $2.905(2.316)$ & 5 & 1.270 \\
\hline constant & 2 & $457(128)$ & $90,526(74,757)$ & $2.904(2.314)$ & 5 & 1.240 \\
delay & 4 & $451(122)$ & $89,616(73,988)$ & $2.877(2.291)$ & 5 & 1.260 \\
& 8 & $449(120)$ & $88,535(73,179)$ & $2.845(2.265)$ & 5 & 1.280 \\
& 16 & $446(117)$ & $85,699(71,112)$ & $2.760(2.200)$ & 5 & 1.800 \\
\hline random & 2 & $457(128)$ & $90,534(74,765)$ & $2.904(2.314)$ & 5 & 1.320 \\
delay & 4 & $455(126)$ & $90,236(74,491)$ & $2.895(2.306)$ & 5 & 1.220 \\
& 8 & $452(123)$ & $89,780(74,115)$ & $2.882(2.295)$ & 5 & 1.230 \\
& 16 & $450(121)$ & $89,299(73,763)$ & $2.868(2.284)$ & 5 & 1.330 \\
\hline probability & 2 & $450(121)$ & $89,490(73,884)$ & $2.873(2.288)$ & 5 & 1.270 \\
& 4 & $429(116)$ & $83,831(69,523)$ & $2.703(2.152)$ & 4 & 1.980 \\
& 8 & $446(117)$ & $85,722(71,136)$ & $2.760(2.201)$ & 5 & 2.020 \\
& 16 & $441(112)$ & $84,637(70,295)$ & $2.728(2.117)$ & 4 & 0.910 \\
\hline limit & 1 & $457(128)$ & $90,590(74,821)$ & $2.906(2.316)$ & 4 & 2.530 \\
simultaneity & 8 & $457(128)$ & $90,514(74,751)$ & $2.903(2.314)$ & 4 & 1.410 \\
\hline no parseBytecode & - & $456(127)$ & $69,463(58,430)$ & $2.248(1.788)$ & 3 & 1.190 \\
\hline openjit-int & - & $329(0)$ & $37,059(31,149)$ & $1.265(0.941)$ & 2 & 1.280 \\
\hline
\end{tabular}
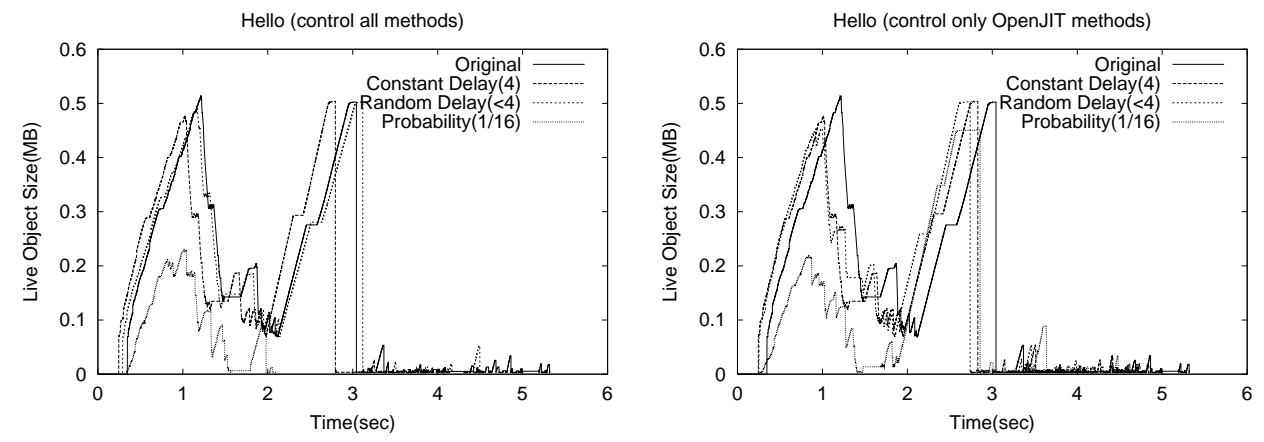

Fig. 10. Timeline behavior of heap usage for adaptive compilation (best cases). 


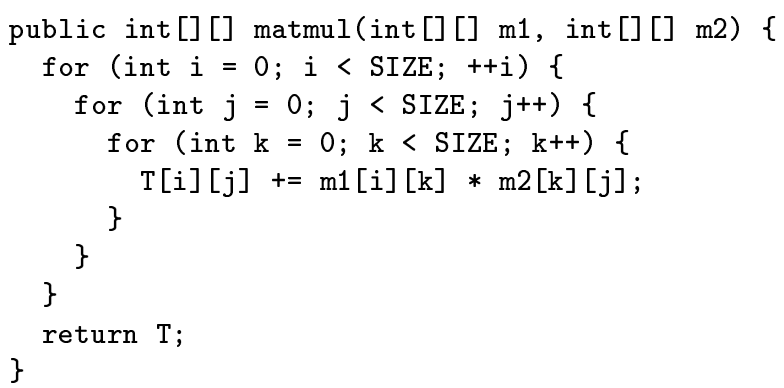

Fig. 12. Matrix Multiply Method (Original)

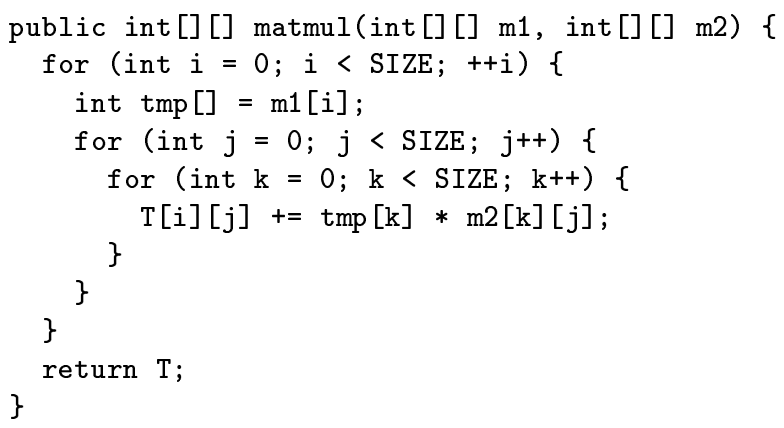

Fig. 13. Matrix Multiply Method (Transformed)

We see that the execution time of OpenJIT and sunwjit are within $10 \%$ of each other. This similar to SpecJVM98 where OpenJIT and sunwjit for SPARCs. So, for the purposes of this benchmark, we can regard both systems to be essentially equivalent, and thus the benefits of reflection can be judged in a straightforward way.

The setup time for OpenJIT without frontend transformation is approximately $1.09 \mathrm{sec}-$ onds, compared to 0.49 seconds for sunwjit. This verifies our benchmarks in the previous section where the compiler bootstrap overhead was quite small. The 1.59 seconds difference between the original and transformed is the overhead of frontend execution. The overhead consists of the process described in Section 3. We believe we can improve this overhead substantially, as the frontend has not been tuned as much as the backend, especially regarding generation of numerous small objects.

Still we see that, although when the matrix size is small $(200 \times 200)$, the overhead of frontend processing with a compilet exceeds that of the speed gain, for larger problem $(600 \times$ 600 ) this overhead is amortized for $7 \%$ improvement. Moreover, we expect to further amortize this as the transformation is done only once, and as a result, multiple execution of the same method will not pay the overhead allowing us to essentially ignore the setup overhead for $9 \%$ gain.

Table 6. Results of OpenJIT Frontend Optimization (All times are seconds)

\begin{tabular}{l|rr|rr}
\hline matrix size & \multicolumn{2}{|c|}{200} & \multicolumn{2}{c}{600} \\
& before & after & before & after \\
\hline OpenJIT & 2.52 & 2.26 & 85.22 & 77.74 \\
OpenJIT setup-time & 1.09 & 2.68 & 1.09 & 2.67 \\
sunwjit & 2.34 & 2.06 & 80.19 & 73.55 \\
sunwjit setup-time & 0.49 & 0.49 & 0.49 & 0.49 \\
\hline
\end{tabular}




\section{Related Work}

As mentioned earlier, most modern compilers and language systems are bootstrapped in a selfdescriptive fashion, but they do not coexist at runtime. In fact, although Lisp and Smalltalk systems embodied their own compilers written in terms of itself and executable at run-time, they are typically source-to-bytecode compilers, and not bytecode-to-native code compilers, which JITs are. In fact, as far as we know, there have not been any reports of a JIT compiler for a particular language being reflective. Most JIT compilers we have investigated, including those for Lisp, Smalltalk, Java as well as experimental languages such as SELF, have been written in $\mathrm{C} / \mathrm{C}++$ or in assembly language.

More recent efforts in self-descriptive, practical object-oriented system is Squeak[IKM $\left.{ }^{+} 97\right]$. Squeak employs the Bluebook[GR83] self-definition of Smalltalk, then bootstraps it using C, then further optimizes the generated VM. Bootstrapping in Squeak involves the VM only, and not the JIT compiler. The recently-announced JIT Squeak compiler is written in C and basically only merges the code fragments corresponding to individual bytecode. Thus, this is not a true compiler in a sense, but rather a simple bytecode to binary translator. This was done to achieve very quick porting of Squeak to various platforms, and stems from some of the earlier work done in [PR98].

We know of only two other related efforts paralleling our research, namely MetaXa[Gol98] and Jalapeño[AAC $\left.{ }^{+}\right]$. Metaxa overall is a comprehensive Java reflective system, constructing a fully reflective system whereby many language features could be reified, including method invocations, variable access, and locking. MetaXa has built its own VM and a JIT compiler; as far as we have communicated with the MetaXa group, their JIT compiler is not full-fledged, and is specific to their own reflective JVM. Moreover, their JIT is not robust enough to compile itself ${ }^{8}$.

Jalapeño[ $\left.\mathrm{AAC}^{+}\right]$is a major IBM effort in implementing a self-descriptive Java system. In fact, Jalapeño is an aggressive effort in building not only the JIT compiler, but the entire JVM in Java.

The fundamental difference stems from the fact that Jalapeño rests on its own customized JVM with completely shared address space, much the same way the C-based JIT compilers are with C-based JVMs. Thus, there is little notion of separation of the JIT compiler and the VM for achieving portability, and the required definition of clean APIs, which is mandated for OpenJIT. For example, the JIT compilers in Jalapeño can access the internal objects of the JVM freely, whereas this is not possible with OpenJIT. So, although OpenJIT did not face the challenges of JVM bootstrapping, this gave rise to investigation of an effective and efficient way of interfacing with a monolithic, existing JITs, resulting in very different technical issues as have been described in Section 4.

The manner in which Jalapeño bootstraps is very similar to Squeak and other past systems. The way the type safety of Java is circumvented, however, is similar to the technique employed in OpenJIT: there is a class called Magic, which defines a set of native methods that implements operations where direct access to VM internals are required. In OpenJIT, the Compile class defines a set of APIs using a similar technique. Unfortunately, again there is no mention of attempting to develop the API into a clean one for generalized purposes of self-descriptive JITs for Jalapeño.

There are other technical differences as well; OpenJIT is architected to be a compiler framework, supporting features such as decompilation, various frontend libraries, whereas it is not with Jalapeño. No performance benchmarks have been made public for Jalapeño, whereas we present detailed studies of execution performance validating the effectiveness of reflective JITs, in particular memory profiling technique which directly exploits the 'openness' of OpenJIT. Interestingly enough, Jalapeño is claimed to be only targeting server platforms,

\footnotetext{
${ }^{8}$ In fact, we are considering collaborative porting of OpenJIT to their system.
} 
and not desktop nor embedded platforms. It would be quite interesting to investigate the memory performance of Jalapeño in the manner we have done, in particular to test whether it makes sense to target smaller platforms or not.

Still, the Jalapeño work is quite impressive, as it has a sophisticated three-level compiler system, and their integrated usage is definitely worth investigating. Moreover, there is a possibility of optimizing the the application together with the runtime system in the VM. This is akin to optimization of reflective systems using the First Futamura projection in object oriented languages, as has been demonstrated by one of the author's older work in [MMAY95] and also in [MY98], but could produce much more practical and interesting results. Such an optimization is more difficult with OpenJIT, although some parts of JVM could be supplanted with Java equivalents, resulting in a hybrid system.

There have been a number of work in practical reflective systems that target Java, such as OpenJava[TC98], Javassist[Chi98], jContractor[KHB99], EPP[IR97], Kava[WS99], just to name a few. Welch and Stroud present a comprehensive survey of Java reflective systems, discussing differences and tradeoffs of where in the Java's execution process reflection should occur[WS99].

Although a number of work in the context of open compilers have stressed the possibility of optimization using reflection such as OpenC $++[$ Chi95], our work is the first to propose a system and a framework in the context of a dynamic (JIT) compiler, where run-time information could be exploited. A related work is Welsh's Jaguar system[WC99], where a JIT compiler is employed to optimize VIA-based communication at runtime in a parallel cluster.

From such a perspective, another related area is dynamic code generation and specialization such as [EP, GPM+99, Fuj]. Their intent is to mostly provide a form of run-time partial evaluation and code specialization based on runtime data and environment. They are typically not structured as a generalized compiler, but have specific libraries to manipulate source structure, and generate code in a "quick" fashion. In this sense they have high commonalities with the OpenJIT frontend system, sans decompilation and being able to handle generalized compilation. It is interesting to investigate whether specialization done with a full-fledged JIT compiler such as OpenJIT would be either be more or less beneficial compared to such specific systems. This not only includes execution times, but also ease of programming for customized compilation. Consel et. al. have investigated a hybrid compile-time and run-time specialization techniques with their Tempo/Harrisa system [VCC, SLCM99], which are source-level Java specialization system written in C; techniques in their systems could be applicable for OpenJIT with some translator to add annotation info for predicated specializations.

\section{Conclusion and Future Work}

We have described our research and experience of designing and implementing OpenJIT, an open-ended reflective JIT compiler framework for Java. In particular, we proposed an architecture for a reflective JIT compiler framework on a monolithic VM, and identify the technical challenges as well as the techniques employed, including the minimal set of low-level APIs required that needed to be added to existing JVMs to implement a JIT compiler in Java, contrasting to similar work such as Jalapeno. We performed analysis of the performance characteristics of OpenJIT, both in terms of execution speed and memory consumption, using collaborative instrumentation technique between the JVM and OpenJIT, which allowed us to instrument the JIT performance in real-time, and showed that OpenJIT is quite competitive with existing, commercial JIT systems, and some drawbacks in memory consumption during the bootstrap process could be circumvented without performance loss. We demonstrate a small example of how reflective JITs could be useful class- or application specific customization and optimization by defining a compilet which allowed us to achieve 8-9\% performance gain without changing the base-level code. 
Numerous future work exists for OpenJIT. We are currently redesigning the backend so that it will be substantially extensible, and better performing. We are also investigating the port of OpenJIT to other systems, including more modern VMs such as Sun's research JVM (formerly EVM). In the due process we are investigating the high-level, generic API for portable interface to VMs. The frontend requires substantial work, including speeding up its various parts as well as adding higher-level programming interfaces. Dynamic loading of not only the compilets, but also the entire OpenJIT system, is also a major goal, for live update and live customization of the OpenJIT. We are also working on several projects using OpenJIT, including a portable DSM system[SOM99], numerical optimizer, and a memory profiler whose early prototype we employed in this work. There are numerous other projects that other people have hinted; we hope to support those projects and keep the development going for the coming years, as open-ended JIT compilers have provided us with more challenges and applications than we had initially foreseen when we started this project two years ago.

\section{References}

$\left[\mathrm{AAC}^{+}\right] \quad$ Bowen Alpern, Dick Attanasio, Anthony Cocchi, Drek Lieber, Stephen Smith, Ton Ngo, and John J Barton. Implementing Jalapeno in Java. In Proceedings of OOPSLA '99.

[ADJ ${ }^{+}$96] Malcom Atkinson, Laurent Daynes, Mick Jordan, Tony Printezis, and Susan Spence. An Orthogonally Persistent Java. ACM SIGMOD Record, 25(4), December 1996.

[AFT99] Yariv Aridor, Michael Factor, and Avi Teperman. cJVM: a Single System Image of a JVM on a Cluster. In Proceedings of International Conference on Parallel Processing '99, September 1999.

[Chi95] Shigeru Chiba. A Metaobject Protocol for C++. In Proceedings of OOPSLA '95, pages 285-299, 1995.

[Chi98] Shigeru Chiba. Javassist - A Reflection-based Programming Wizard for Java. In Proceedings of OOPSLA'98 Workshop on Reflective Programming in C++ and Java, October 1998.

[DH99] Sylvia Dieckman and Urs Holzle. A Study of the Allocation Behavior of the SPECjvm98 Java Benchmarks. In Proceedings of ECOOP '99, pages 92-115, 1999.

[EP] Dawson R. Engler and Todd A. Proebsting. vcode: a retargetable, extensible, very fast dynamic ocde generation system. In Proceedings of PLDI' 96.

[Fuj] Nobuhisa Fujinami. Automatic and Efficient Run-Time Code Generation Using ObjectOriented Languages. In Proceedings of ISCOPE '97, December.

[Gol98] Michael Golm. metaXa and the Futre of Reflection. In Proceedings of OOPSLA'98 Workshop on Reflective Programming in C++ and Java, October 1998.

$\left[\mathrm{GPM}^{+} 99\right] \quad$ B. Grant, M. Philipose, M. Mock, C. Chambers, and S. Eggers. An Evaluation of Staged Run-time Optimization in DyC. In Proceedings of PLDI '99, 1999.

[GR83] Adele Goldberg and David Robson. Smalltalk-80: The Language and Its Implementation. Addison-Wesley, Reading, MA, 1983.

[Hol95] Hrs Holzle. Adaptive optimization for Self: Reconciling High Performance with Exploratory Programming. Technical Report STAN-CS-TR-94-1520, Stanford CSD, 1995.

$\left[\mathrm{IKM}^{+} 97\right]$ Dan Ingalls, Ted Kaehler, John Maloney, Scott Wallace, and Alan Kay. Back to the Future: The Story of Squeak - A Usable Small talk Written in Itself. In Proceedings of OOPSLA '97, pages 318-326, October 1997.

[IR97] Yuuji Ichisugi and Yves Roudier. Extensible Java Preprocessor Kit and Tiny DataParallel Java. In Proceedings of ISCOPE '97, December 1997.

[KHB99] Murat Karaorman, Urs Holzle, and Joh Bruno. iContractor: A Reflective Java Library to Support Design by Contract. In Proceedings of Reflection '99, pages 175-196, July 1999.

$\left[\mathrm{KLM}^{+} 97\right]$ Gregor Kiczales, John Lamping, Anurag Mendhekar, Chris Maeda, Cristina Lopes, JeanMarc Loingtier, and John Irwin. Aspect-Oriented Programming. In Proceedings of ECOOP ' ' 7 , pages 220-242, 1997. 
[MMAY95] Hidehiko Masuhara, Satoshi Matsuoka, Kenichi Asai, and Akinori Yonezawa. Compiling Away the Meta-Level in Object-Oriented Concurrent Reflective Languages Using Partial Evaluation. In Proceedings of OOPSLA '95, pages 57-64, October 1995.

[MOM99] Fuyuhiko Maruyama, Hirotaka Ogawa, and Satoshi Matsuoka. An Effective Decompilation Algorithm for Java Bytecodes. IPSJ Journal PRO (written in Japanese), 1999.

[MOS ${ }^{+98}$ Satoshi Matsuoka, Hirotaka Ogawa, Kouya Shimura, Yasunori Kimura, and Koichiro Hotta. OpenJIT - A Reflective Java JIT Compiler. In Proceedings of OOPSLA'98 Workshop on Reflective Programming in C++ and Java, October 1998.

[MY98] Hidehiko Masuhara and Akinori Yonezawa. Design and Partial Evaluation of Metaobjects for a Concurrent Reflective Language. In Proceedings of ECOOP '98, pages 418-439, July 1998.

[Ole97] Ole Agesen. Design and Implementation of Pep, a Java Just-In-Time Translator. Theory and Practice of Object Systems, 3(2):127-155, 1997.

[PR98] Ian Piumarta and Fabio Riccardi. Optimizing Direct-threaded Code by Selective Inlining. In ACM SIGPLAN Conference on Programming Language Design and Implementation (PLDI '98), pages 291-300, June 1998.

[Shi98] Kouya Shimura. OpenJIT Backend Compiler. http://www.openjit.org/docs/backendcompiler/openjit-shimura-doc-1.pdf, June 1998.

[SK96] Kouya Shimura and Yasunori Kimura. Experimental development of java jit compiler. In IPSJ SIG Notes 96-ARC-120, pages 37-42, October 1996.

[SLCM99] Ulrik Pagh Schultz, Julia L. Lawall, Charles Consel, and Gilles Muller. Towards Automatic Specialization of Java Programs. In Proceedings of ECOOP '99, June 1999.

[SM99] Kouya Shimura and Satoshi Matsuoka. OpenJIT Backend Compiler (Runtime) Internal Specification version 1.1.7. http://www.openjit.org/docs/backend-internal/index.html, October 1999.

[SOM99] Yukihiko Sohda, Hirotaka Ogawa, and Satoshi Matsuoka. OMPC++ - A Portable High-Performance Implementation of DSM using OpenC++ Reflection. In Proceedings of Reflection '99, pages 215-234, July 1999.

[TC98] Michiaki Tatsubori and Shigeru Chiba. Programming Support of Design Patterns with Compile-time Reflection. In Proceedings of OOPSLA'98 Workshop on Reflective Programming in $\mathrm{C}++$ and Java, October 1998.

[TS97] Todd Proebsting and Scott Watterson. Krakatoa: Decompilation in Java. In Proceedings of COOTS '97, June 1997.

[Uni] Stanford University. SUIF Homepage. http://www-suif.stanford.edu/.

[VCC] Eugen N. Volanschi, Charles Consel, and Crispin Cowan. Declarative Specialization of Object-Oriented Programs. In Proceedings of OOPSLA '97, pages 286-300, October.

[WC99] Matt Welsh and David Culler. Jaguar: Enabling Efficient Communication and I/O from Java. Concurrency: Practice and Experience, December 1999. Special Issue on Java for High-Performance Applications.

[WS99] Ian Welch and Robert Stroud. From Dalang to Kava - the Evolution of a Reflective Java Extention. In Proceedings of Reflection '99, pages 2-21, July 1999. 